\title{
EARTH LEAKAGE PROBLEM OF SUPERCONDUCTOR MAGNET SYSTEM AT TLS
}

\author{
Yuan-Chen Chien, Kou-Hwa Hu, Kuo-Bin Liu, Hsu-Ming Shih \\ NSRRC, Hsin-Chu, Taiwan
}

\section{Abstract}

Due to the inherent connection structure between the power supplies and the superconducting magnetic coil, the power supplies are seeing each others as loads through the coil. It has been observed in the pilot test at TLS that there is about $300 \mathrm{~mA}$ earth leakage current flowing through the earth leakage protection circuit, which presented an interlock and halted the whole system down. The existence of earth leakage current may undermine the overall system stability and pose more threat on the electronic system safety. In this paper, the earth leakage mechanisms are thoroughly examined and measures to conquer the problem are proposed.

\section{INTRODUCTION}

Several superconducting devices have been installed in the recent years in NSRRC. There are already SWLS, SW6, IARW-R6 in service right now, which provide quality beam lines for various applications. Two more IARW superconducting devices are to be expected in the end of 2007. While the success of superconducting devices, during the pilot run phase, current earth leakage problem was observed and posed threat to the system safety and stability.

A typical superconducting magnet system is depicted in figure 1 .

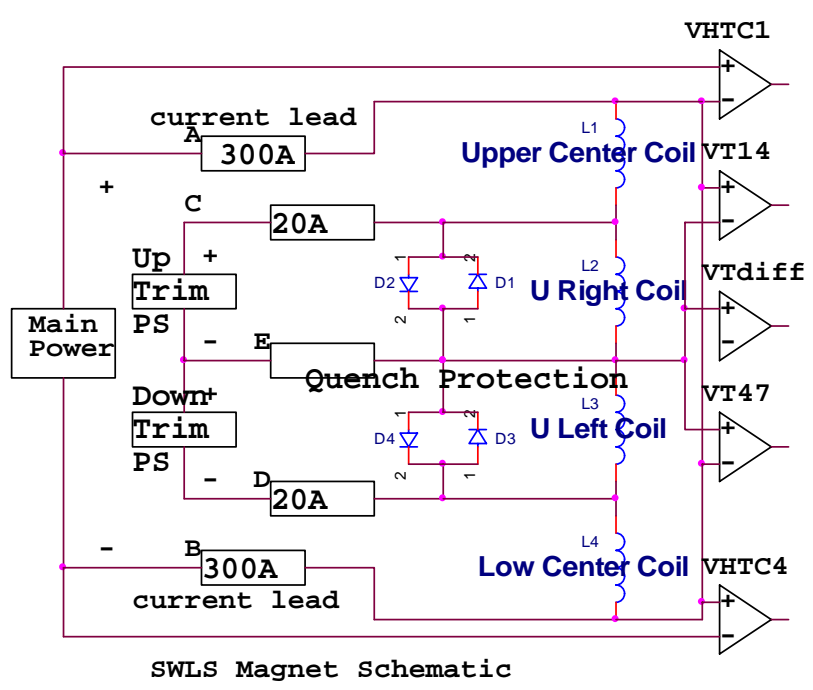

Figure 1: Superconducting Magnet System Diagram

In this diagram, the main power supply is a bipolar 350A/15V constant current source, which will provide the major magnet field energy. Besides, there are two 20A trim power supplies attached in the up-stream and down- stream coil respectively. There are also five voltage monitor points connected directly to the main coil.

Due to the near-zero resistance property of the superconducting magnet coil, all the power supplies and monitor devices connected to the coil are transparent to each others. Because of this, cares must be taken to isolate each device connected to the coil, otherwise current may branch its ways and causes earth leakage fault on the main power supply.

\section{EARTH LEAKAGE SPIKE}

In the very beginning after setting up the system, the whole system failed to function due to main power supply trip off due to earth leakage. The earth leakage detector converts the leakage current to voltage signal and then the voltage output Vleakage is connected to a voltage level controller which will trip off the power supply if Vleakage is greater than $1 \mathrm{~V}$, which is equivalent to $10 \mathrm{~mA}$ of earth leakage. Figure 2 shows the measured leakage current Ileakage and Vleakage. The Ileakage and Vleakage can go as high as $300 \mathrm{~mA}$ and $30 \mathrm{~V}$ respectively before the level controller trip off the supply.

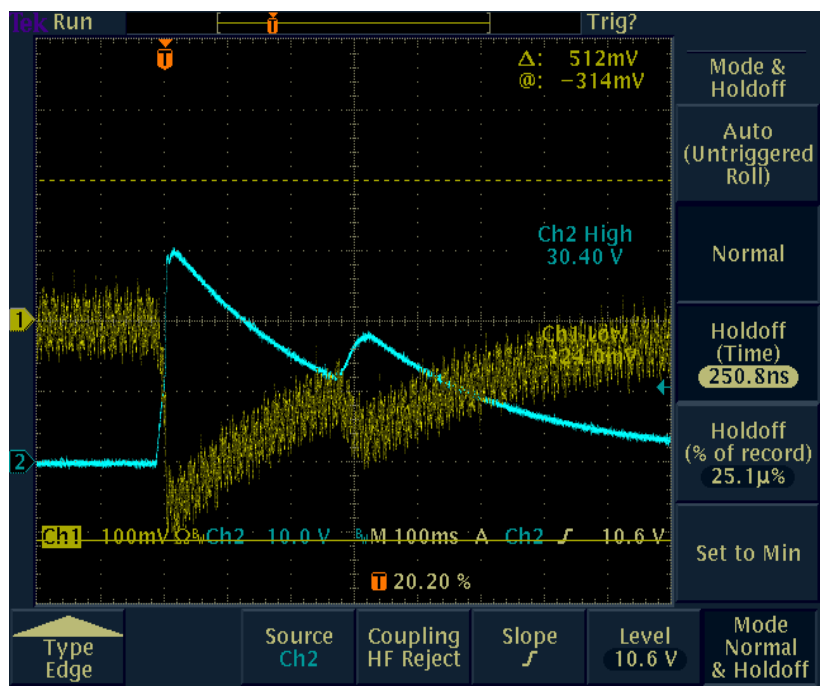

Figure 2: Measured Aggregated Ileakage and Vleakage

The CH1 yellow line and $\mathrm{CH} 2$ blue line represents the Ileakage and Vleakage respectively.

\section{LEAKAGE CURRENT LOOPS}

By careful examination of the overall system connection diagram, four current loops are identified as culprits which cause the earth leakage problem. These current loops as depicted in figure 3 are : 

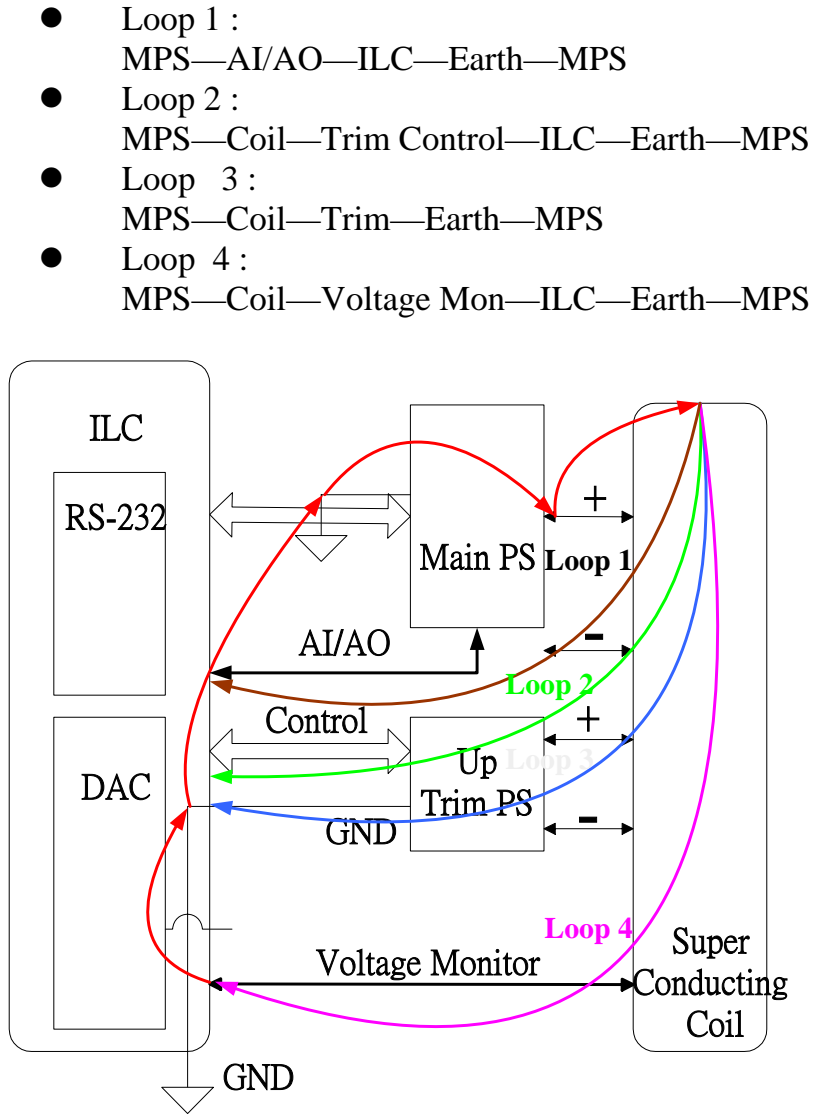

Figure 3: The power supply ground loop in the SWLS system

A. Loop1: The main power current leaks through the main power supply's AI command input and AO current read back to the ILC , then the current leak goes through the common earth and back to the main power supply .

B. Loop 2 : This loop path is from the coil to the trim power's output cables and then goes all the way back to main power by the no-isolated trim power control signals from ILC unit.

C. Loop 3 : Loop 3 is similar to Loop 2 , but the leak path goes directly through the trim-earth contact to the main power instead of the trim power control lines.

D. Loop 4 : The current leaks through the 5 coil voltage tapped out monitor points. This loops path is formed cause the tapped out points are connected right to the coil without any isolation inside the superconducting chamber.

In order to sort out each current loop's contribution to the aggregated earth leakage current, only one loop leakage current is measured while the others three are broken. The procedures are repeated till all the leakage current of the four loops are measured. The measured leakage current for each current loop is listed in Table 1.
Table 1: Measured leakage current

\begin{tabular}{|l|c|}
\hline Loop & Earth Leakage Current in $\mathbf{~ A A}$ \\
\hline 1 & 250 \\
\hline 2 & 230 \\
\hline 3 & 300 \\
\hline 4 & 184 \\
\hline
\end{tabular}

As can be seen from the Table 1 listed above, Loop 1, 2, 3 except loop 4 contributes sufficient portion of leakage current to the aggregated earth leakage current. The leakage current measured in current loop 4 is showed in figure 4.

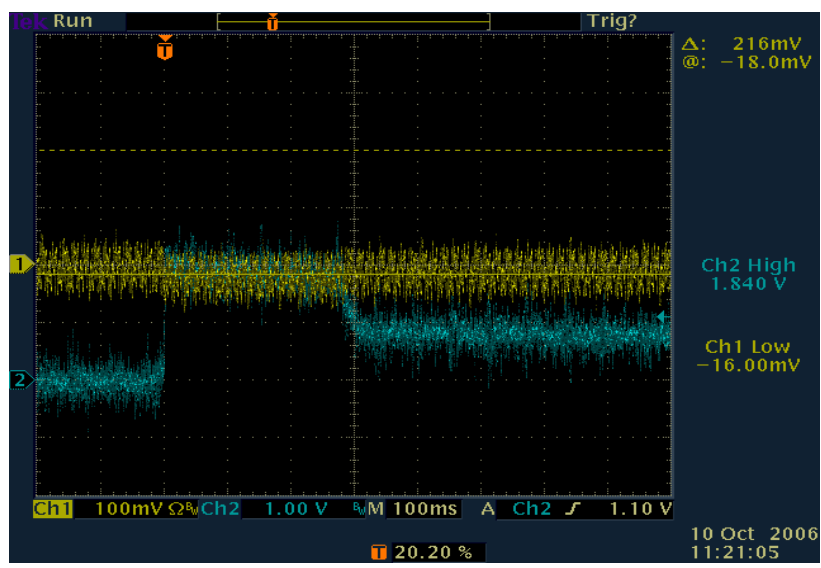

Figure 4: Loop 4 Ileakage and Vleakage

\section{LOOP BREAKING STRATEGIES}

The earth leakage current problem may manifest itself in

A. Improper choose of trim power supplies with nonfloating full isolated output stage.

B. Non-isolated analogue command in/current out to the power supplies from ILC unit.

C. Improper isolated Voltage monitor read back.

In case A can easily be solved by employing power supplies with full isolated output stage with same output rating.

For case B, the earth leakage current is caused by nonisolated analogue single-ended command input and current readback to/from the power supplies. As seen from the figure 3 , the analogue command inputs to the two trim power supplies come from a DAC board, while the current readback are fed into the ADC board embedded in the ILC unit (Intelligent Logic Control). For both the command signal and current readback are singleended, the ground of the DAC/ADC boards are connected through the power supply's ground and then to the main power supply ground. This constitutes ground loops and hence introduce earth leakage problem. This problem can be solved by 
1) Employing power supplies with isolated I/O buffers built-inside or

2) Feeding the DAC/ADC I/O to an isolation board which converts the single-ended signals into differential signal pairs and then connecting the differential pairs to the power supplies.

Strategy 2 is used here to cut the ground loops even the power supplies do not have fully-isolated I/O buffers. All the I/O to and from the ILC unit must all be isolated electrically. Isolation amplifier or opto-coupler is proper candidate for this situation. Here with isolation and precision taken into account, isolation amplifier ISO124 modules are inserted to all the $\mathrm{I} / \mathrm{O}$ lines. The photo of the ISo124 isolation module is shown in figure 5.

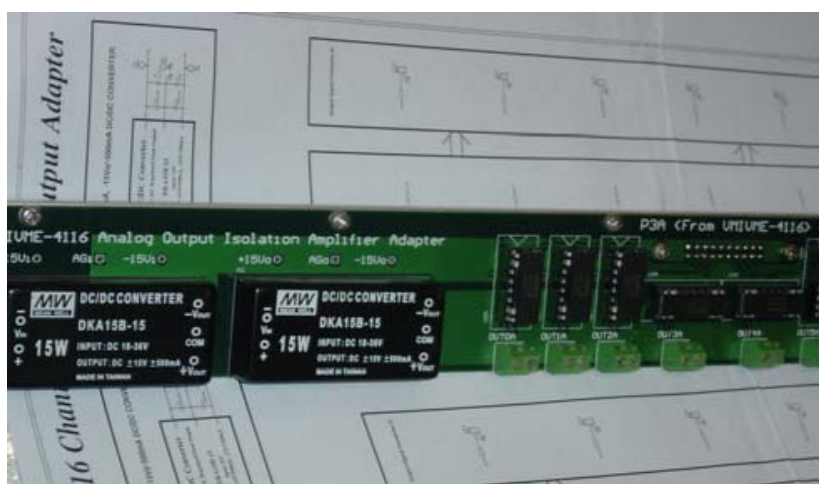

Figure 5 : I/O Isolation Module
The same method for Case B is also applied in the last case too. Previously, INA117 has been attempted before the coil voltage signals enter the ADC board in the ILC. Because INA117 is not fully electrically isolation amplifier, this type of buffer cannot deliver acceptable isolating performance.

\section{CONCLUSION}

By carefully examining all the interconnections inside the superconducting magnet system, four current loops which lead to the main power supply's earth leakage problem are identified. Isolation strategies have been taken to conquer this leakage problem with satisfying success. Breaking the current loops does not only enhance the power supplies' operating stability and performance, but most importantly, guarantee the safety of the overall superconducting magnet system.

\section{REFERENCES}

[1] Yuan-Chen Chien, K.T. Hsu, C.S. Hwang, C.Y.Liu, K.B. Liu, "Stability Study of Superconductor Magnet Power Supplies at TLS “, EPAC, 2006.

[2] Yuan-Chen Chien, "A Report On Earth Leakage Current of Superconductor Magnet System”, NSRRC, Internal Report, Dec 2006. 